\title{
Bingineering Jourtinal
}

Article

\section{Three-Dimensional Data Quality Assessment: Unmanned Aerial Vehicle Photogrammetry and Mobile Laser Scanner}

\author{
Mohd Azwan Abbas ${ }^{1, a, *}$, Khairulazhar Zainuddin ${ }^{1, b}$, Norhadija Darwin ${ }^{2, \mathrm{c}}$, \\ Mohamad Aizat Asyraff Mohamad Azmi ${ }^{1, d}$, Yusuf Drisu Opaluwa ${ }^{3, e}$, \\ Saiful Aman Sulaiman ${ }^{1,4, \mathrm{f}}$, and Norshahrizan Mohd Hashim ${ }^{1, g}$ \\ 1 Centre of Studies for Surveying Science \& Geomatics, Universiti Teknologi MARA Perlis Branch, Arau \\ Campus, 02600 Arau, Perlis, Malaysia \\ 2 Faculty of Built Environment \& Surveying, Universiti Teknologi Malaysia, 81310 Skudai, Malaysia \\ 3 Department of Surveying \& Geoinformatics, School of Environmental Technology, Federal University of \\ Technology Minna, Nigeria \\ 4 Malaysia Institute of Transport (MITRANS), Universiti Teknologi MARA, 40450 Shah Alam, Selangor, \\ Malaysia \\ E-mail: amohdazwan@uitm.edu.my (Corresponding author), bkhairul760@uitm.edu.my, \\ cnorhadija2@utm.my, ${ }^{\mathrm{d}}$ sraizatazmi@gmail.com, ${ }^{\mathrm{e}}$ opaluwayd@futminna.edu.ng, fsaifulaman@uitm.edu.my \\ gnorshahrizan@uitm.edu.my
}

\begin{abstract}
With significantly rapid in acquiring data and sufficient data quality, unmanned aerial vehicle (UAV) photogrammetry and land based mobile laser scanner are actively implemented in three-dimensional (3D) data acquisition that involve with large area. Considering the final data provided by both measurement approaches are point clouds, accuracy assessment using several well-distributed control points are less significant. With intention to robustly evaluate the accuracies of both measurement approaches using point clouds, this study has established reference point clouds using terrestrial laser scanner (TLS) and tacheometry techniques. At the similar test site, fourteen (14) images were captured using UAV photogrammetry approach and georeferenced point clouds were directly acquired from MLS measurement. To produce quality point clouds from photogrammetry approach, six (6) ground control points (GCP) have been well-distributed at the test area to aid geometry correction in image processing phase. Obtained point clouds from both measurement approaches were deviated with the reference point clouds to determine values of mean deviations with the precisions. Based on law of propagation of variance (LOPOV) algorithm, final accuracy of the tested UAV photogrammetry and MLS were computed by propagating the accuracy of reference point clouds and yielded mean deviations of both approaches. Consider the theories and constraints for both approaches, it is found that the yielded accuracies are meet the measurement principles.
\end{abstract}

Keywords: Unmanned aerial vehicle, photogrammetry, laser scanner, tacheometry, surface deviation, accuracy assessment.

ENGINEERING JOURNAL Volume 25 Issue 1

Received 9 June 2020

Accepted 25 November 2020

Published 31 January 2021

Online at https:/ / engj.org/

DOI:10.4186/ej.2021.25.1.143 


\section{Introduction}

Requirement of three-dimensional (3D) data acquisition has been highly demand in many applications which related to documentation, management, analysis and decision making. Applications that require 3D information are cultural heritage [1, 2], 3D city database [3], structural deformation measurements [4, 5], stability analysis for hazardous natural features [6], slope monitoring [7] and industrial measurements $[8,9]$. In geomatic jargon, there are plenty of sensors capable to provide $3 \mathrm{D}$ data such as tacheometry, global navigation satellite system (GNSS), photogrammetry and LiDAR (light detection and ranging). Due to the measurement mechanism employed by tacheometry and GNSS which oblige single point observation at a time has become a constraint in providing dense 3D points. These methods can be considered as time consuming and cumbersome (during data collection phase), also most of the time failed to provide the amount of details required [10]. In contrast, photogrammetry and LiDAR measurement approaches has the capability to yield dense 3D data with significantly rapid acquiring procedure. According to Wolf et al. [11], photogrammetry technique has been classified under two types which are terrestrial (handheld camera or mounted to a tripod) and aerial (camera mounted on the unmanned aerial vehicle, airplane or satellite). In order to obtain $3 \mathrm{D}$ data from twodimensional (2D) images, extensive manual editing and refinement is essential [12]. On the other hand, whether through terrestrial or aerial approaches, LiDAR measurement technique able to directly provide 3D data. Though, mounting LiDAR sensor to a mobile platform has made this sensor dependence on other positioning techniques (i.e. GNSS and inertial measurement unit) to determine the position and direction of LiDAR device [13].

Bureaucracy in acquiring data can expose the findings to uncertainties, which eventually can decrease the quality of final products. With the aid of structure-from-motion (SfM) algorithm, unmanned aerial vehicle (UAV) photogrammetry has been widely utilised for many purposes including forestry and agriculture, archaeology and cultural heritage, environmental surveying, traffic monitoring and 3D reconstruction [14]. However, requirement of extensive processing to yield dense 3D point clouds has expose this measurement approach with propagation of errors. Similar situation happen to mobile LiDAR (mobile laser scanner) measurement when derivation of final 3D point clouds are dependence on quality of GNSS and IMU devices to determine the moving sensor.

Currently, accuracy assessment for both UAV photogrammetry and mobile laser scanner (MLS) were examined based on several control points that have been well-distributed at site [15-17]. Taking into account the dense 3D data (point clouds) provided by both measurement approaches, this assessment method only capable to averagely measure the quality of the acquired data. All points yielded should be included in the evaluation to concretely verify the quality of the measurement. In other word, the assessment is quite unbalance when dense $3 \mathrm{D}$ points were deviated with benchmark which consisted of few reference points. With the existence of static LiDAR (terrestrial laser scanner) and the capability to provide sub-centimetre level of accuracy [18], it is possible to robustly evaluate the all dense points yielded from UAV photogrammetry and mobile LiDAR. Furthermore, Luhmann et al. [19] have stated that terrestrial laser scanner (TLS) has better accuracy than aerial photogrammetry (Fig. 1) and considering propagation of errors from other sensors, it is undeniable that TLS is superior to MLS.

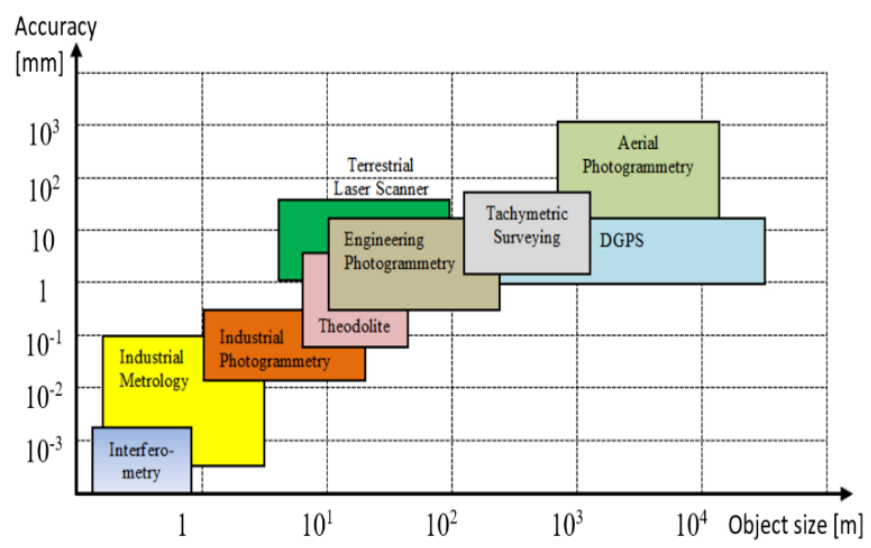

Fig. 1. Accuracy of the sensors with respect to the object sizes [19].

With intention to critically verify the accuracy of UAV photogrammetry and mobile laser scanner, this study has exploited TLS data as reference point clouds. Beforehand, the quality of TLS has been measured to ensure the reliability to become benchmark for this study. To fairly examine the accuracy, all yielded points from UAV photogrammetry and MLS have been utilised in surface deviation analysis. Law of propagation of variances algorithm was employed to mathematically determine the accuracy of both measurements (i.e. UAV photogrammetry and MLS) based on computed precision obtained.

\section{UAV Photogrammetry}

Unmanned Aerial Vehicle (UAV) known as drone which no pilot on board. Capability to fly up to $300 \mathrm{~m}$ with significantly less in operational cost, simple manipulation, flexibility and high-resolution data has made UAV widely used in many applications such as military, mapping and monitoring. Theoretically, the UAV system is equipped with devices such as sensor (e.g. camera, LiDAR or thermal sensor), navigation devices and communication tools.

According to Zongian [20], the advantages in developing the technology of UAV for low altitude photogrammetric mapping are to perform aerial photography under the cloud, to get full image of object from the top, and to supply a cheap and easy system for 
high frequency needs of aerial photogrammetric survey. In addition, Eisenbeiss [21] did mentioned that UAV photogrammetry can be understood as a new photogrammetric measurement tool and opens various new applications in the close-range domain, combining aerial and terrestrial photogrammetry.

In photogrammetric micro UAV, other than UAV (with sensors) itself, ground station is also crucial element that need to take into account. This station can aid UAV pilot to perform multiple tasks including data acquisition and measurement, servo driving, automatic flight control implementation, communications and data logging. The ground station allows the user to aware about GPS status, battery voltage, and communication link status. Figure 2 show the Phantom 4 UAV with ground controller (station) utilised in this study.

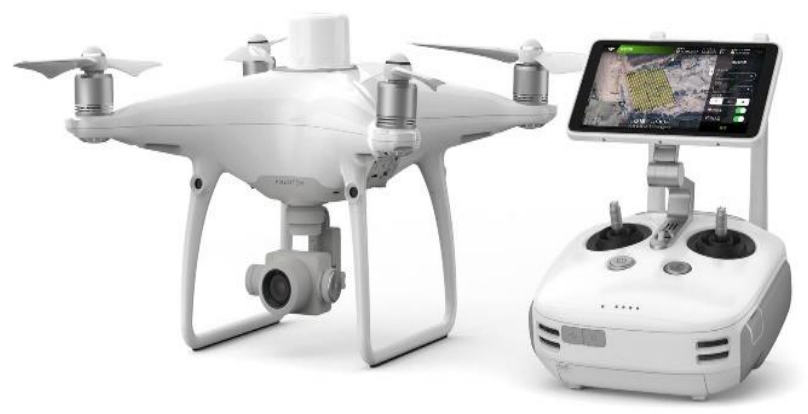

Fig. 2. Phantom 4 UAV with ground controller.

There were two main outputs of photogrammetric produced after through several steps of digital image processing such as digital elevation model (DEM) and orthophoto. Orthophoto is an aerial photograph which is geometrically corrected (orthorectified), lacks of lens distortion, camera tilt and an accurate representation of the earth's surface [22]. Meanwhile, DTM represents the earth surface or part of the earth surface digitally. The DTM is the most important element in any mapping purposes which consist of mathematical representation of the ground [23]. According to Tahar [24], the accuracy of DTM is influenced by the density of control points that are used in generating the DTM. It can be concluded that, the number of control points influence the accuracy and resolution of DTM. However, the quality of control points that are used to generate DTM depends on the accuracy of ground measurement.

\section{Land Based LiDAR System}

Light detection and Ranging (LiDAR) technology has been widely used in mobile mapping system (MMS). The mechanism adopted which directly measure range between sensor and targeted object, provides a significant improvement in term of data density and accuracy with respect to the conventional data acquisition approaches. For land-based implementation, LiDAR was exploited for static and mobile measurement. The crucial issues that have differentiate both approaches are registration and georeferencing procedures. As discussed in Abbas et al. [25], static LiDAR (also known as terrestrial laser scanner) only require minimum three (3) well-distributed targets (artificial or natural) measure by pairwise scanners (as depicted by distribution of spheres in Fig. 3) to enable several local coordinate systems (based on number of occupied stations) to be oriented into one global coordinate system. As georeferencing procedure also employ similar coordinate transformation algorithm (Eq. 1), requirement of well-distributed targets are analogous except it did not demand for pairwise condition as registration procedure (sphere targets with tripod in Fig. 3).

$$
X_{i}=T+S \cdot R \cdot x_{i}
$$

where:

$$
\begin{aligned}
\mathrm{X}_{\mathrm{i}}= & \text { Coordinates of the ith target in the } \\
& \text { reference (or global) scanner coordinate } \\
& \text { system (Xi, Yi and } \mathrm{Zi}) . \\
\mathrm{S}= & \text { Scale factor. } \\
\mathrm{R}= & \text { Components of rotation matrix } \\
& \text { between the two coordinate systems } \\
& (\omega, \varphi, x) .
\end{aligned}
$$

Determine final position for Land based mobile LiDAR or mobile laser scanner (MLS) is quite complicated. Due to the used of mobile platform, scanner position should be in homogenous coordinate system. Most of the LiDAR system provided by the manufacturer is equipped with global navigation satellite system (GNSS) receiver, inertial navigation system (INS) for acceleration and orientation measurements of the moving platform and, in most cases, a wheel-mounted Distance

Measuring Indicator (DMI), which provides accurate vehicle velocity updates [26]. To improve the positioning accuracy, rather than stand-alone GNSS, MLS has been augmented with real time kinematic (RTK) GNSS which has accuracy of centimetres level [27-29]. Figure 4 illustrated the positioning system utilise in MLS measurement.

Both land-based LiDAR approaches have shown significant contribution in acquiring dense $3 \mathrm{D}$ data. Capability to perform measurement in mobile platform has made MLS able to acquire data in large area. In contrast static approach adopted by TLS has limited the measurement coverage due to the data acquisition methodology and time constraint. Complimenting each approach, Dursun et al. [31] have utilised TLSs and MLS in $3 \mathrm{D}$ city modelling, where MLS was employed to map entire city, while TLSs were used to capture complex features from building facades. When it involves with level of details four (LOD4) or demanding data of building interior, thus, TLS is indispensable. Taking into account about the data quality, it is mathematically significant that TLS is superior to MLS. When it goes to errors 
propagation, TLS only expose to measurement uncertainties and algorithm exploited for pre-processing procedure. For MLS, other than errors adopted by TLS, there are also uncertainties contribute from GNSS and INS observations. Puente et al. [26] did mentioned that the accuracy of the MLS instrument is on the order of few centimetres, the overall accuracy of any integrated MLS system is often determined by the accuracy of the navigation solution. According to Ali [27], accuracy of GNSS RTK are $0.030 \mathrm{~m}$ for horizontal and $0.060 \mathrm{~m}$ for vertical. Based on that, it is expected that accuracy for MLS should be within several up to tenths of centimetres. In contrast, accuracy of TLSs have been statistically proven by Abbas et al. [32] are within sub-centimetre level.

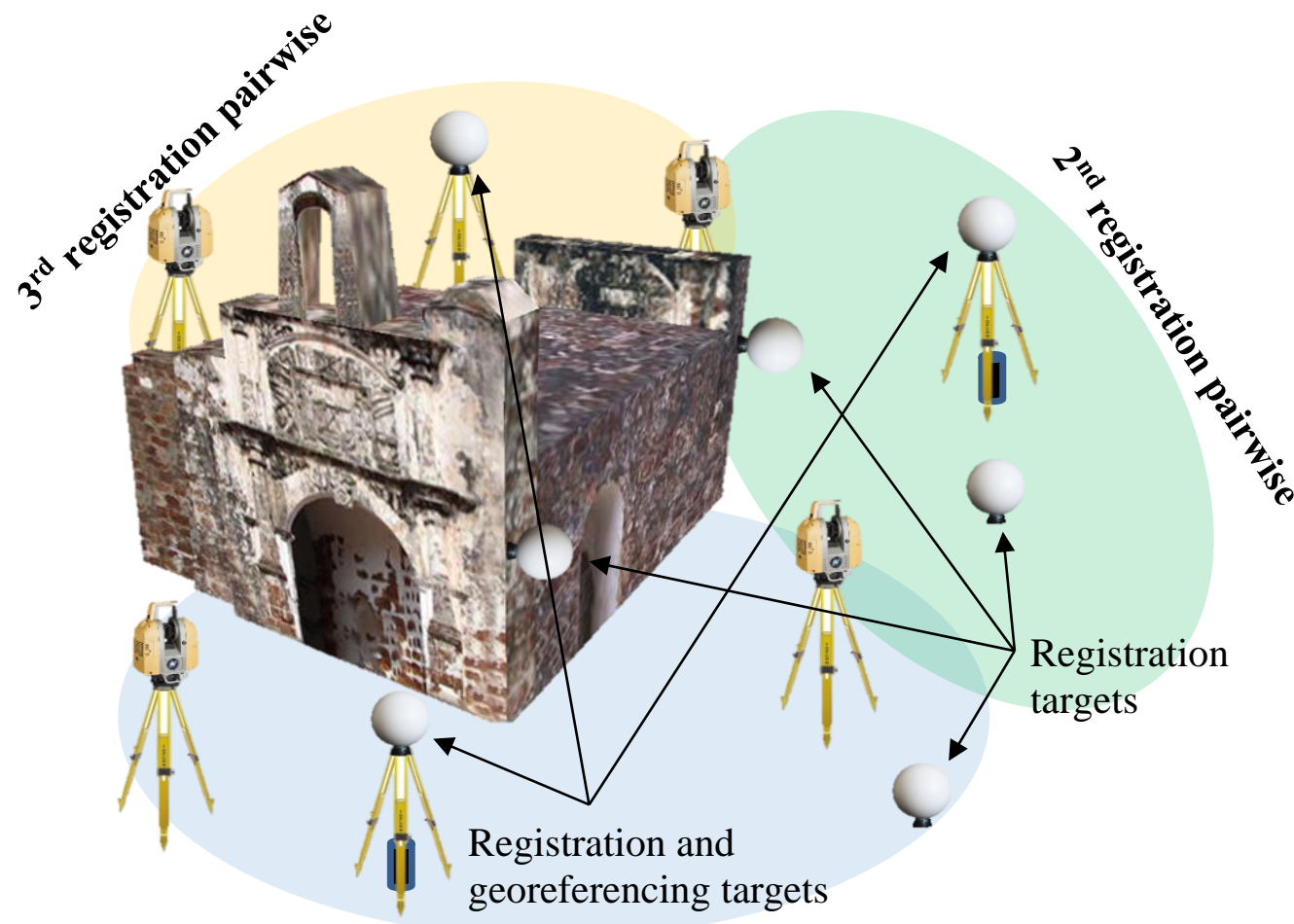

$1^{\text {st }}$ registration pairwise

Fig. 3. Registration and georeferencing procedures for terrestrial laser scanner.

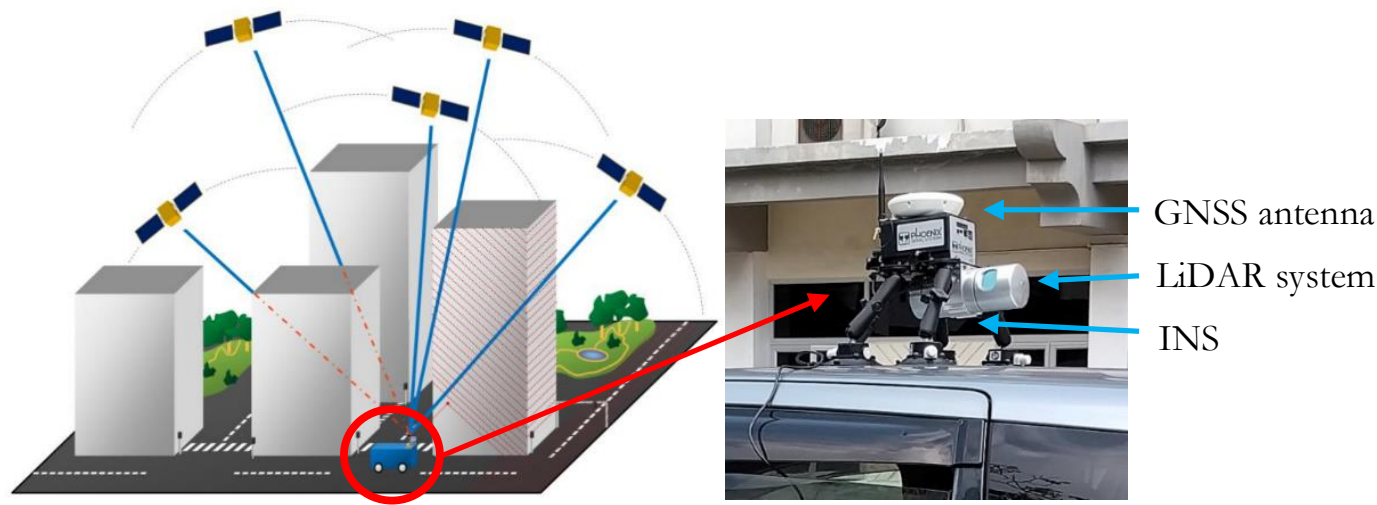

Fig. 4. Mobile laser scanning positioning system [30].

\section{Experiment}

There are four phases of experiments that have been carried out in order to measure the accuracy of point clouds obtained from UAV photogrammetry and MLS measurements. Experiments were initial with establishment of benchmarking points using tacheometry measurement. Based on the results of first phase, quality of reference point clouds (established using TLS measurement) were measured. Third phase focuses on image processing based on the data obtained from UAV photogrammetry. Due to the nature of MLS data, did not require any pre-processing procedure to yield point clouds, thus, final phase discusses on accuracy assessment of clouds provided by UAV photogrammetry and MLS measurements using statistical analysis. 


\subsection{Preparation of Test Points}

As depicted in Fig. 1, Luhmann et al. [19] have found that tacheometry capable to provide data with millimetres level of accuracy. Based on that, sixteen (16) number of artificial targets have been well-distributed and at the test field (Fig. 5). Two occupied tacheometry stations have been utilised to measure all targets using three- dimensional triangulation method. For this study, Topcon ES-105 with accuracy of $0.0014^{\circ}$ and $2 \mathrm{~mm}$ for angular and range measurement, respectively has been utilised. To robustly adjust the data, linear regression algorithm [33] has been employed to yield most probable value of all targets with the observation quality.

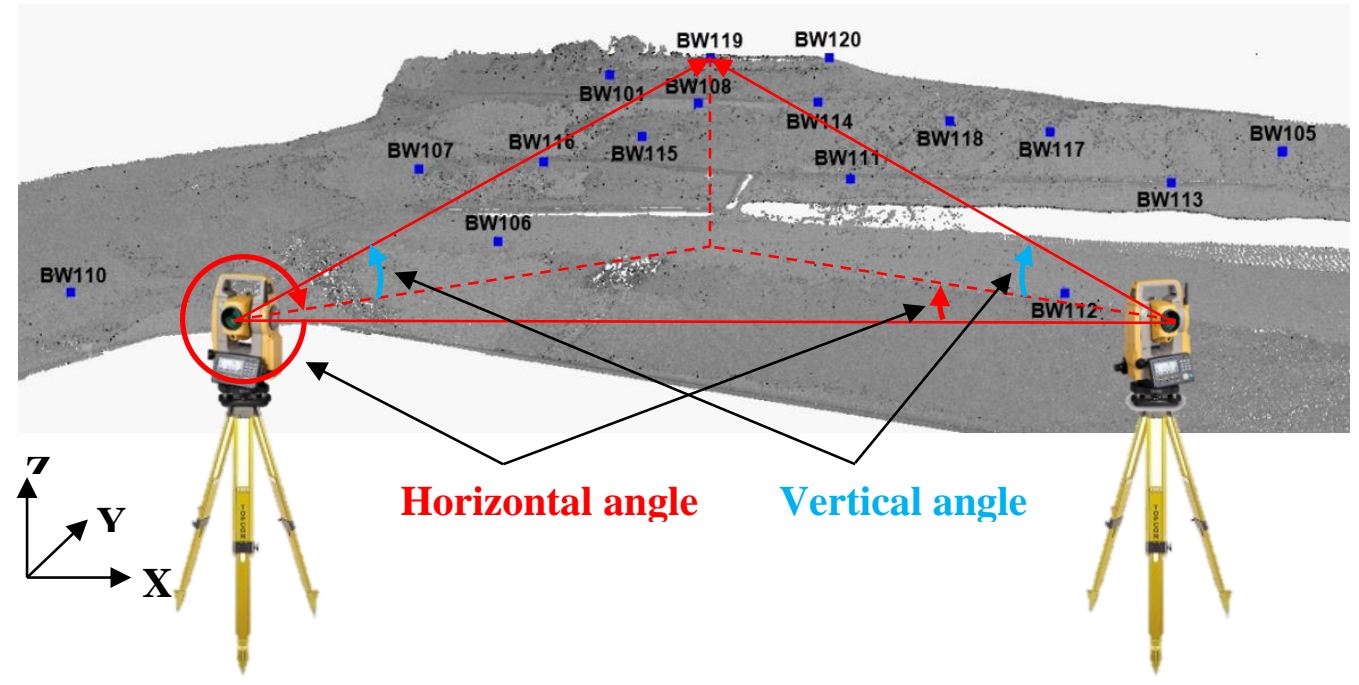

Fig. 5. Sixteen (16) artificial targets measured from two tacheometry positions.

\subsection{Preparation of Reference Point Clouds}

With the aid of reference points established using tacheometry method, reference point clouds were produce using Topcon GLS-2000 scanner. All targets with the surface of the land slope have been scanned from two scanner positions, which have roughly occupied similar as tacheometry positions. According to the instrument specification sheet, this time-of-flight scanner was employed panoramic field-of-view to capture $360^{\circ}$ of horizontal and $270^{\circ}$ of vertical coverage. The accuracies of single point measurement are $3.5 \mathrm{~mm}$ and $0.0017^{\circ}$ for range and angular measurements, respectively. To examine the accuracy of reference point clouds (obtained from Topcon GLS-2000 scanner), geometrical and points analyses have been performed based on the reference points established by tacheometry measurement. Geometrical analysis were carried out by forming fifteen (15) independent vectors from sixteen (16) targets. Geometry discrepancies resulted from the comparison of vectors produced from tacheometry and TLS can be used to measure the accuracy of TLS through statistical formula [33].

Quality of TLS data, $\sigma_{T L S}=\frac{\sum_{i=1}^{n}\left(l_{T L S}-l_{\text {Tacheo }}\right)^{2}}{n}$

Where $n, l_{T L S}$ and $l_{\text {Tacheo }}$ are number of observations, TLS and tacheometry vectors, respectively. Later analysis were performed with the aid of a rigid body transformation as described by Eq. (1). The idea is to mathematically match the 3D points yielded from tacheometry (benchmark) and TLS data. To perform point to point analysis, Australis V6.06 software is used to implement rigid body transformation and subsequently calculate the root mean square (RMS) of the differences between control (tacheometry) and transformed coordinates (TLS). Magnitude of RMS obtained from transformation adjustment will indicate the quality of TLS measurement.

\subsection{UAV Photogrammetry and Mobile Laser Scanner}

Three-dimensional point clouds of the test site from UAV photogrammetry have been yielded from fourteen (14) images captured using UAV built-in camera (refer Fig. 6). UAV utilised in this study is DJI Phantom 4 (rotary wing UAV) which has been equipped with 12.4 megapixel digital camera and positioning device (stand-alone GNSS). To increase the accuracy of the processed point clouds, six (6) visible reference points established by tacheometry have been used as ground control points (GCP), while the rest eight (8) targets were adopted as check points (CP). GCPs were employed as aided for geometry correction in image processing to ensure the quality of yielded map, while CPs play a role to measure the accuracy of the derived DEM model from the generated point cloud.

Agisoft PhotoScan Profesional software was exploited to perform aerial image processing. There were several steps of producing point clouds from UAV photogrammetry which consists of aligning images, 
building model geometry and model texture. Few conditions need to take into account when acquiring data using UAV photogrammetry: 1) High resolution digital camera (minimum five megapixels) with wide angle lens; 2) Fly under the cloud; and 3) Seventy percent aerial images overlapping. In addition, appropriate camera calibration parameters was entered during digital images processing.

To measure the quality of the processed data from UAV photogrammetry, correlation of pixel image and ground should be prior computed. Ground sampling distance (GSD) for digital camera is calculated with the following formula as shown in Eq. (3).

$$
G S D=\frac{h_{g}}{c_{k}} \times C C D
$$

where,

$$
\begin{array}{ll}
h_{g} & =\text { flying height above ground } \\
c_{k} & =\text { focal length } \\
C C D & =\text { pixel size }
\end{array}
$$

The value of the GSD is significant to exemplify the expected accuracy of the by-product such as digital elevation model (DEM) and orthophoto. As stated by Barry and Coakley [34] and Gonçalves and Henriques [35], the rule-of-thumb for the accuracy is vary between two (2) and three (3) times of GSD value.

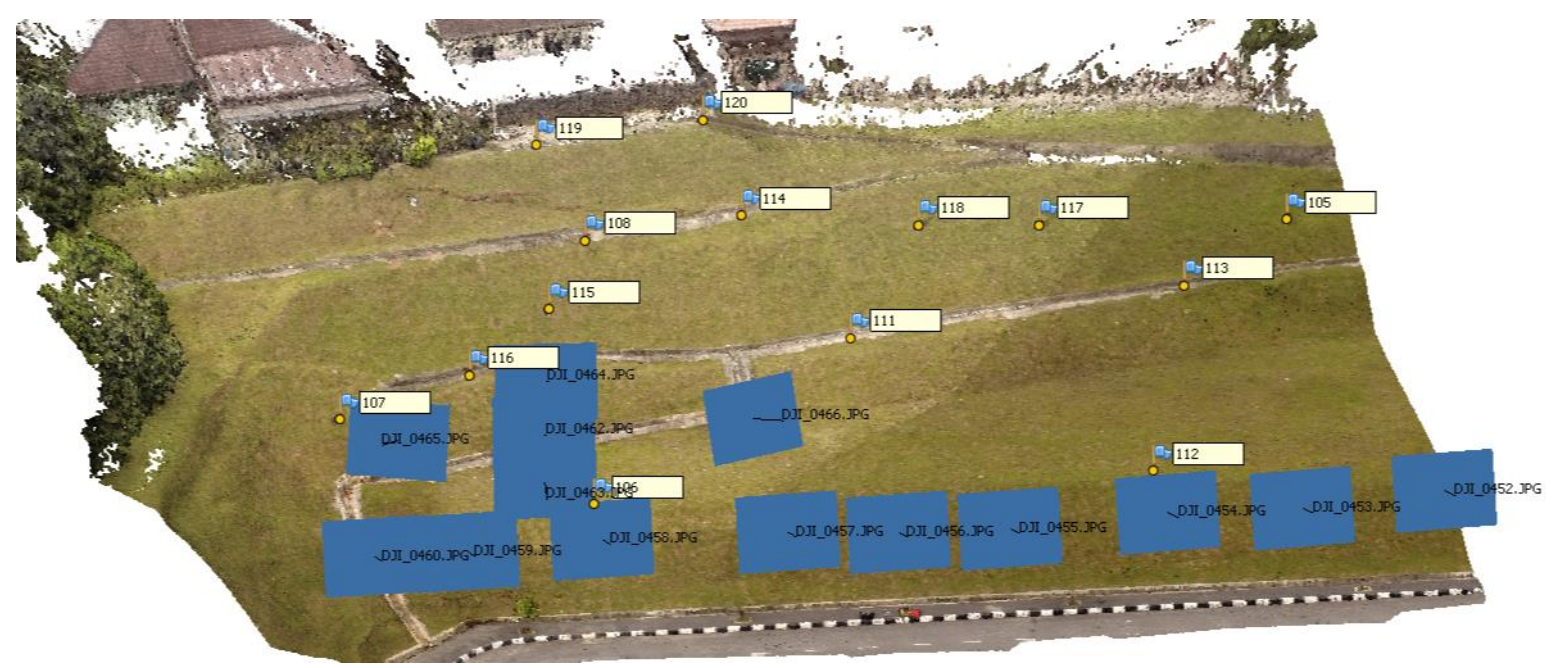

Fig. 6. Fourteen (14) images captured from DJI Phantom 4 camera.

\subsection{Point Clouds Assessment}

For this study, Phoenix AL3 system has been adopted for mobile laser scanner measurement. This scanner capable to measure distance up to $107 \mathrm{~m}$ with 700,000 shots per second. To ensure that scanned data properly covered the whole surface of the test area, two modes of observations were used, which are using vehicle based (Fig. 7) and human based. Equipped with INS and GNSS devices, all point clouds scanned by the Phoenix AL3 system are already registered and georeferenced. Furthermore, with capability to perform real time kinematic (RTK) GNSS measurement to determine the LiDAR system position, Phoenix AL3 able to improve the accuracy of the obtained data. In contrast with TLS and UAV photogrammetry approaches, MLS did not require any extensive processing procedure to yield the final 3D data. For data quality assessment, MLS data has been deviate with reference point clouds produced from TLS measurement.

To finalise the accuracy of both UAV photogrammetry and MLS point clouds, law of propagation of variance (LOPOV) algorithm has been employed. LOPOV is essential to propagate mean precision obtained from reference surface into mean standard deviation yielded from deviation analysis. Absolute accuracy of both measurement approaches from this LOPOV formula [33]:

Accuracy of UAV Photogrammetry or MLS,

$$
\sigma_{\text {Photo } / M L S}=\sqrt{\sigma_{T L S}^{2}+\sigma_{S D}^{2}}
$$

where, $\sigma_{T L S}^{2}$ and $\sigma_{S D}^{2}$ are variance of TLS measurement and mean variance from surface deviation analysis, respectively.

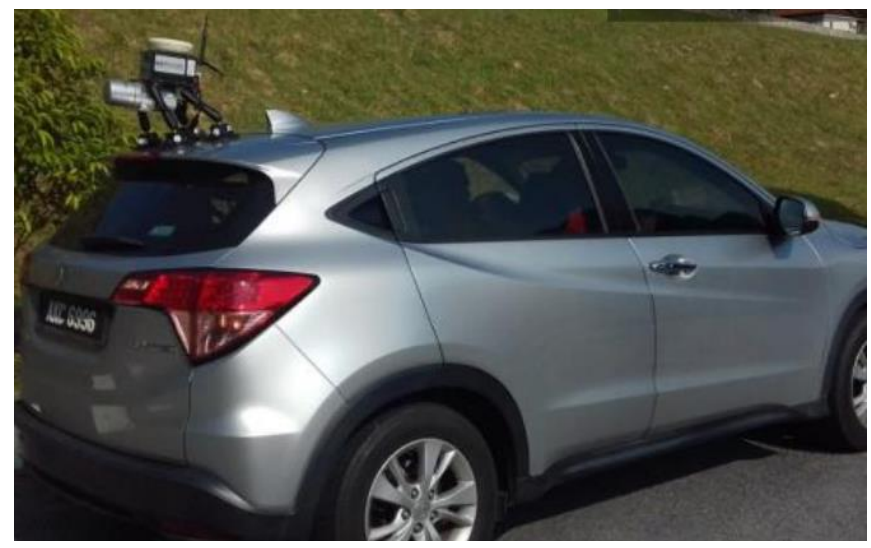

Fig. 7. Vehicle based mobile laser scanning. 


\section{Results and Analyses}

Linear regression outcomes from tacheometry observation to establish sixteen (16) control points have indicated that all data (i.e. horizontal and vertical angles) manage to converge at third iteration and passed global test at 95\% confidence interval. According to Table 1, all targets averagely manage to acquire $\pm 1.4 \mathrm{~mm}$ of standard deviation with maximum values contribute by target BW110. As visualised in Fig. 8, largest error ellipse yielded by target BW110 occurred due to the target position has caused high incidence angle from occupied sensors (S1 and S2 stations). Based on that factor, intersection measurement from two stations has instigated the magnitude of point dilution of precision. Nevertheless, with less than $2 \mathrm{~mm}$ data quality for average standard deviation, adjusted coordinates acquired from tacheometry observation are sufficient to become benchmark for TLS accuracy assessment.

To evaluate the accuracy of Topcon GLS-2000 scanner data acquisition, fifteen independent vectors were established from sixteen (16) targets. Fixing target BW111 as centre point, calculated vectors for both tacheometry and terrestrial laser scanner were organised in Table 2. Based on law of propagation of variance algorithm, it is found that the accuracy of Topcon GLS-2000 scanner is $6 \mathrm{~mm}$. As expected, under 95\% confidence interval (two sigma), accuracy of the scanner as stated by manufacturer would be $7 \mathrm{~mm}$.

Table 1. Adjusted parameters with standard deviation obtained from linear regression adjustment.

\begin{tabular}{ccccccc}
\hline Station & $\mathbf{X}(\mathbf{m})$ & $\mathbf{Y}(\mathbf{m})$ & $\mathbf{Z}(\mathbf{m})$ & $\mathbf{\pm} \boldsymbol{\sigma}_{\mathbf{X}}(\mathbf{m})$ & $\mathbf{\pm} \sigma_{\mathbf{Y}}(\mathbf{m})$ & $\pm \sigma_{\mathbf{Z}}(\mathbf{m})$ \\
\hline S1 & 286935.704 & 597191.494 & 27.446 & 0.000 & 0.000 & 0.000 \\
S2 & 286962.422 & 597249.604 & 28.019 & 0.000 & 0.000 & 0.000 \\
BW110 & 286924.570 & 597179.383 & 29.604 & 0.004 & 0.004 & 0.001 \\
BW102 & 286891.378 & 597189.070 & 40.195 & 0.003 & 0.001 & 0.002 \\
BW101 & 286895.173 & 597201.772 & 38.774 & 0.002 & 0.001 & 0.002 \\
BW119 & 286894.363 & 597209.394 & 40.009 & 0.002 & 0.001 & 0.002 \\
BW120 & 286895.923 & 597218.214 & 40.040 & 0.001 & 0.001 & 0.002 \\
BW108 & 286900.516 & 597208.686 & 37.050 & 0.001 & 0.001 & 0.002 \\
BW114 & 286902.017 & 597216.946 & 37.197 & 0.001 & 0.001 & 0.002 \\
BW118 & 286905.864 & 597225.129 & 36.251 & 0.001 & 0.001 & 0.002 \\
BW107 & 286906.818 & 597190.764 & 33.216 & 0.002 & 0.001 & 0.002 \\
BW116 & 286905.948 & 597198.763 & 33.629 & 0.002 & 0.001 & 0.002 \\
BW115 & 286903.370 & 597204.965 & 35.043 & 0.001 & 0.001 & 0.002 \\
BW106 & 286918.769 & 597198.742 & 30.992 & 0.001 & 0.001 & 0.001 \\
BW111 & 286910.880 & 597218.259 & 33.271 & 0.001 & 0.001 & 0.002 \\
BW104 & 286909.335 & 597248.560 & 37.494 & 0.001 & 0.001 & 0.002 \\
BW103 & 286919.773 & 597268.882 & 36.276 & 0.002 & 0.001 & 0.002 \\
BW105 & 286912.383 & 597242.872 & 35.112 & 0.001 & 0.001 & 0.002 \\
BW113 & 286914.106 & 597235.892 & 33.558 & 0.001 & 0.001 & 0.002 \\
BW117 & 286908.025 & 597230.880 & 35.802 & 0.001 & 0.001 & 0.002 \\
BW112 & 286930.310 & 597224.884 & 31.286 & 0.001 & 0.001 & 0.001 \\
BW109 & 286947.826 & 597255.439 & 30.308 & 0.001 & 0.001 & 0.001 \\
\hline
\end{tabular}

Table 2. Independent vectors for TLS accuracy assessment.

\begin{tabular}{cccc}
\hline Vector & Tacheometry $(\mathbf{m})$ & TLS $(\mathbf{m})$ & Discrepancies $(\mathbf{m})$ \\
\hline BW111 - BW101 & 23.427 & 23.424 & 0.003 \\
BW111 - BW105 & 24.727 & 24.729 & 0.001 \\
BW111 - BW106 & 21.174 & 21.177 & 0.003 \\
BW111 - BW107 & 27.793 & 27.792 & 0.001 \\
BW111 - BW108 & 14.607 & 14.610 & 0.003 \\
BW111 - BW110 & 41.379 & 41.359 & 0.020 \\
BW111 - BW101 & 20.624 & 20.622 & 0.002 \\
BW111 - BW105 & 17.927 & 17.927 & 0.000 \\
BW111 - BW112 & 9.783 & 9.786 & 0.003 \\
BW111 - BW113 & 15.371 & 15.371 & 0.000 \\
BW111 - BW114 & 20.113 & 20.110 & 0.003 \\
BW111 - BW115 & 13.186 & 13.194 & 0.009 \\
BW111 - BW116 & 9.013 & 9.012 & 0.002 \\
BW111 - BW117 & 19.920 & 19.917 & 0.003 \\
BW111 - BW118 & 16.417 & 16.415 & 0.002 \\
\hline
\end{tabular}




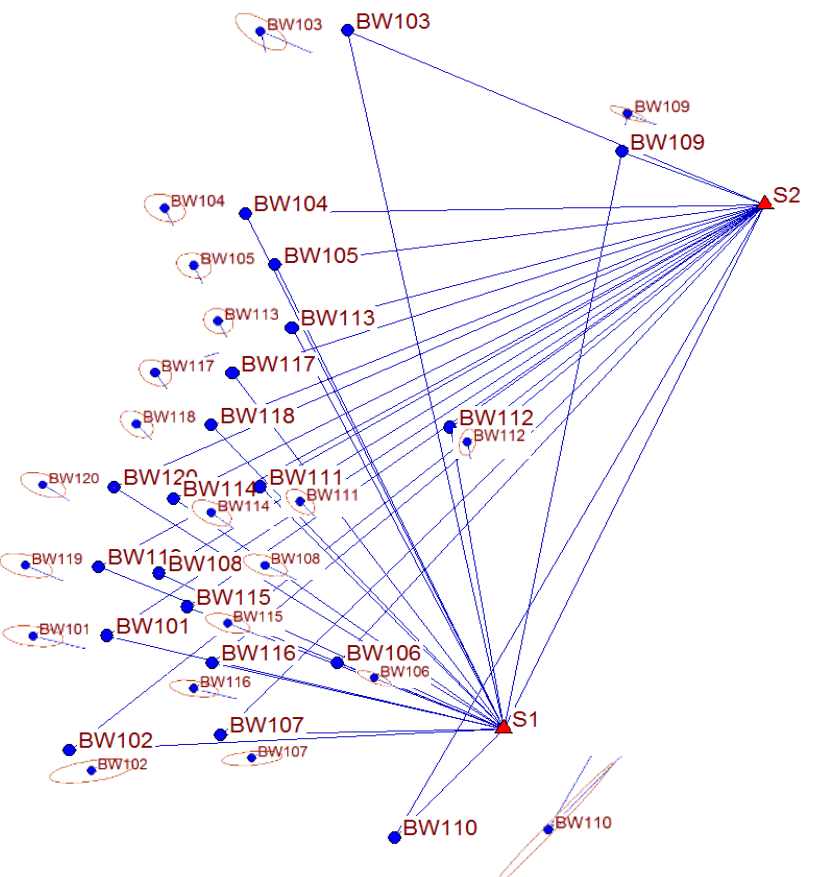

According to Fig. 9, variation of three-dimensional coordinates $(\mathrm{X}, \mathrm{Y}$ and $\mathrm{Z}$ ) discrepancies are quite large, from $0.1 \mathrm{~mm}$ up to $19.5 \mathrm{~mm}$. As expected, largest discrepancy was contributed by target BW110. To fairly examine differences of points yielded from both measurement, rigid body transformation algorithm was employed to perform coordinates transformation adjustment. After fourth iterations, RMS of differences for both systems (i.e. tacheometry and TLS) are $0.004 \mathrm{~m}$, $0.008 \mathrm{~m}$ and $0.003 \mathrm{~m}$ for $\mathrm{X}, \mathrm{Y}$ and $\mathrm{Z}$ axes, respectively. Similar to geometrical analysis finding, computed RMS of all axes has demonstrated that point to point analysis also manage to acquire $6 \mathrm{~mm}$ data quality. Consider the accuracy of UAV photogrammetry and MLS, which are centimetres level, thus, 3D data obtained from Topcon GLS-2000 scanner is sufficient to become reference point clouds.

Fig. 8. Plotted error ellipses for the adjusted coordinates of artificial targets.

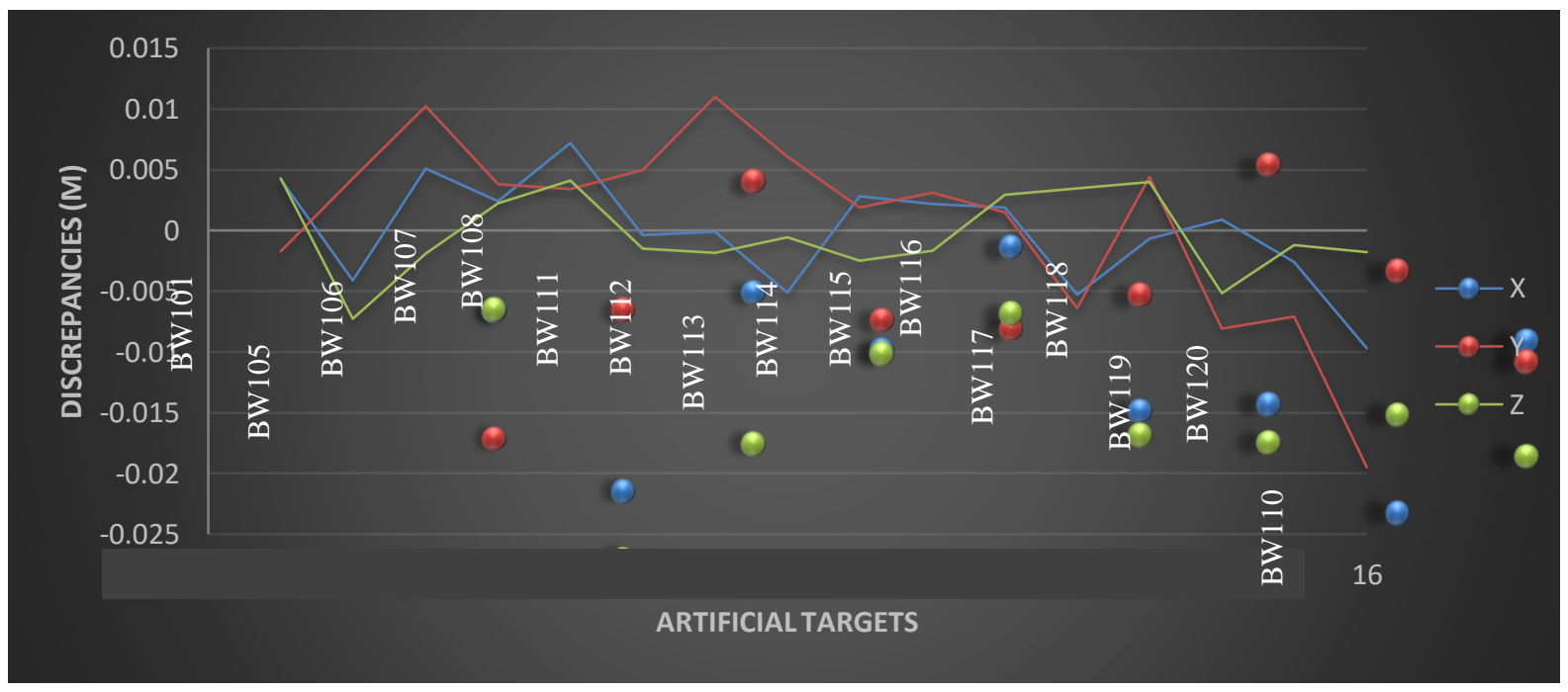

Fig. 9. Three-dimensional coordinates discrepancies of tacheometry and TLS data.

For the UAV photogrammetry processing, the report has shown that the 3D model of UAV imagery at altitude of $40 \mathrm{~m}$ yielded an average of $1.09 \mathrm{~cm}$ per pixel ground sampling distance (GSD). Using the pre-calibrated camera value, the DSM was derived from 4,300 sparse point clouds with the re-projection error of 0.469 pixel, indicates the quality of the 3D reconstruction from image have an error less than 1-pixel size (or sub pixel accuracy). As mentioned earlier, six (6) targets were used as GCP and the rest eight (8) targets were utilized as check point (CP). Exemplified in Table 3 and Table 4, outcomes from the image processing demonstrated that RMSE obtained are $0.871 \mathrm{~cm}$ and $1.028 \mathrm{~cm}$, for GCPs and CPs, respectively.
The largest error of point marking on GCPs given by the target BW112, which have only projected by six images with error of $1.22 \mathrm{~cm}$. While the largest error for $\mathrm{CPs}$ given by target BW105 with error magnitude of $2.14 \mathrm{~cm}$. Based on the image processing results, the quality of the $3 \mathrm{D}$ reconstruction gives the error less than $2 \mathrm{GSD}$ (i.e. $1.09 \mathrm{~cm}$ per pixel GSD). Considering the principle of photogrammetry where the accuracy of aerial photogrammetry is vary between two (2) and three (3) times of GSD value, thus, the processed point clouds from UAV photogrammetry (for this study) should lie within two (2) to three (3) centimetre. 
Table 3. Errors report for GCPs.

\begin{tabular}{cccccc}
\hline Targets & X error $\mathbf{( c m )}$ & Y error $(\mathbf{c m})$ & Z error $(\mathbf{c m})$ & XYZ $(\mathbf{c m})$ & Image (pixel) \\
\hline BW119 & -0.754 & -0.118 & 0.415 & 0.869 & $0.292(13)$ \\
BW107 & 0.320 & -0.060 & -1.033 & 1.083 & $0.218(11)$ \\
BW115 & 0.018 & -0.307 & -0.304 & 0.432 & $0.311(12)$ \\
BW106 & -0.346 & 0.078 & 0.301 & 0.465 & $0.179(9)$ \\
BW117 & 0.593 & 0.607 & -0.116 & 0.857 & $0.285(11)$ \\
BW112 & -0.653 & 0.898 & 0.507 & 1.220 & $0.187(6)$ \\
Total & 0.511 & 0.464 & 0.531 & 0.871 & 0.260 \\
\hline
\end{tabular}

Table 4. Errors report for CPs.

\begin{tabular}{cccccc}
\hline Targets & X error $\mathbf{( c m )}$ & Y error $\mathbf{( c m )}$ & Z error $(\mathbf{c m})$ & XYZ $\mathbf{( c m )}$ & Image (pixel) \\
\hline BW120 & -0.778 & 0.239 & 0.514 & 0.962 & $0.296(14)$ \\
BW108 & -0.186 & -0.028 & 0.327 & 0.377 & $0.295(13)$ \\
BW114 & -0.115 & 0.288 & 0.440 & 0.538 & $0.385(9)$ \\
BW118 & 0.070 & -0.385 & 0.249 & 0.464 & $0.339(12)$ \\
BW116 & 0.673 & -0.049 & -0.283 & 0.732 & $0.270(12)$ \\
BW111 & 0.159 & 0.092 & 0.423 & 0.461 & $0.408(10)$ \\
BW105 & 1.259 & -1.543 & -0.803 & 2.148 & $0.277(5)$ \\
BW113 & 1.026 & -0.452 & -0.512 & 1.232 & $0.271(3)$ \\
Total & 0.687 & 0.601 & 0.473 & 1.028 & 0.325 \\
\hline
\end{tabular}

With the assistance of CloudCompare open source software, point clouds acquired from UAV photogrammetry and MLS have been deviated with reference point clouds (obtained from TLS). Figure 10 visualised the result from surface deviation analysis of UAV photogrammetry and TLS, while Fig. 11 is deviation outcome from MLS and TLS. As illustrated in Fig. 10, cloud to cloud deviations for UAV photogrammetry and TLS are quite homogenous, colour scale discrepancies are not very significant with mean deviation is $0.023 \mathrm{~m}$. In contrast, clouds deviation of MLS and TLS which yield mean deviation of $0.067 \mathrm{~m}$ have demonstrated a substantial colour scale differences, especially at the slope area (red circle). The situation occurred may be due to the limitation of GNSS measurement constraint, where satellites geometry have disadvantage in determine elevation element as stated in
Ali [27]. Computed standard deviations yielded from analyses are $\pm 0.029 \mathrm{~m}$ and $\pm 0.060 \mathrm{~m}$ for UAV photogrammetry and MLS, respectively. Propagating those deviation errors with TLS error (i.e. $0.006 \mathrm{~m}$ ) using LOPOV algorithm, the result still produce similar errors. In other word, the accuracy of point clouds produced by UAV photogrammetry and MLS are $\pm 0.029 \mathrm{~m}$ and $\pm 0.060 \mathrm{~m}$, respectively. As discussed earlier, based on aerial photogrammetry principle, it is expected that UAV photogrammetry point clouds manage to provide accuracy up to three (3) centimetre. For the MLS point clouds, it is also expected when the system dependence on other sensors to determine the position. With centimetres level of accuracy for RTK GNSS observation, taking into account INS and MLS measurement errors, six (6) centimetre accuracy for MLS data is very realistic.

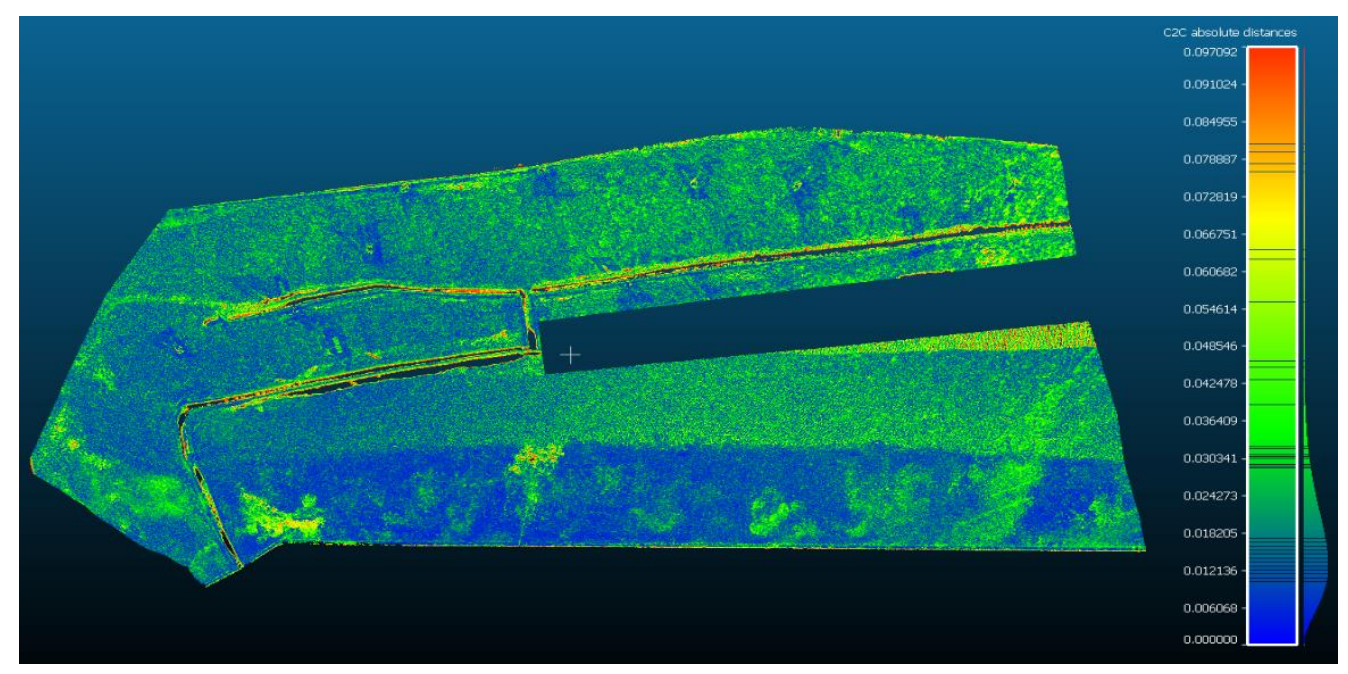

Fig. 10. Surface deviation of UAV photogrammetry and reference point clouds. 


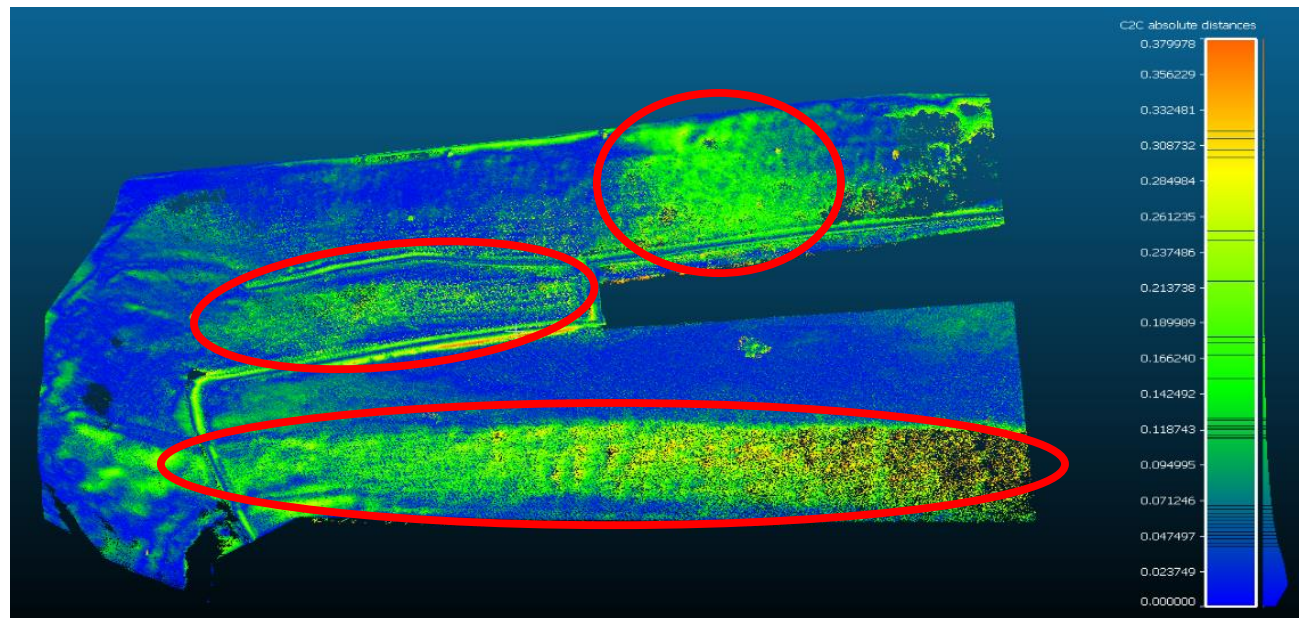

Fig. 11. Surface deviation of MLS and reference point clouds.

\section{Conclusions}

With the aim to robustly measure the accuracy of point clouds obtained from UAV photogrammetry and MLS measurements, this study has employed TLS and tacheometry approaches to establish reference point clouds. Beforehand, a rigid procedure has been held in order to examine the accuracy of reference point clouds. Sixteen (16) artificial targets were well-distributed and measured using tacheometry and TLS techniques. With the aid of statistical analysis, is it found that the yielded reference point clouds manage to achieve accuracy of $6 \mathrm{~mm}$. Observation using UAV photogrammetry and MLS have properly designed to ensure the quality of the final outcome. For the UAV photogrammetry, six (6) welldistributed GCPs have been utilised to rectify the geometry of the yielded map. Furthermore, eight (8) CPs were used to measure the accuracy of obtained point clouds. While MLS measurement has exploited the capability of RTK GNSS, instead of utilising stand-alone GNSS. To ensure that all surfaces covered by MLS, two different measurement mode have been carried out, which are vehicle and human based. Having point clouds from both measurement approaches, surface deviation analyses were performed and the computed precisions were used to calculate the accuracy. Through LOPOV algorithm, the final accuracies of UAV photogrammetry and MLS have demonstrated outcomes that are adhere to the measurement principle.

\section{Acknowledgement}

We would like to thank to Universiti Teknologi MARA for the financial funding. Special thanks goes to Nursyahira Ahmad Fuad, Anuar Aspuri and Mohd Faizi Mohd Salleh for the aid during data acquisition and processing. Last but not least, my gratitude to Photogrammetry and Laser Scanning Research Group, Universiti Teknologi Malaysia for providing instruments and experiment site.

\section{References}

[1] G. Tucci, G. Bartoli, M. Betti, V. Bonora, M. Korumaz, and A. G. Korumaz, "Advanced procedure for documenting and assessment of cultural heritage: From laser scanning to finite element," IOP Conference Series: Materials Science and Engineering, vol. 364, no. 1, p. 012085, 2018.

[2] R. K. Napolitano, G. Scherer, and B. Glisic, "Virtual tours and informational modeling for conservation of cultural heritage sites," Journal of Cultural Heritage, vol. 29, pp. 123-129, 2018.

[3] B. Bonczak and C. E. Kontokosta, "Large-scale parameterization of 3D building morphology in complex urban landscapes using aerial lidar and city administrative data," Computers, Environment and Urban Systems, vol. 73, pp. 126-142, 2018.

[4] X. Xu, H. Yang, and I. Neumann, "Deformation monitoring of typical composite structures based on terrestrial laser scanning technology," Composite Structures, vol. 202, pp. 77-81, 2018.

[5] H. Yang, X. Xu, and I. Neumann, "Optimal finite element model with response surface methodology for concrete based on terrestrial laser scanning technology," Composite Structures, vol. 183, pp. 2-6, 2018.

[6] R. Kromer, M. Lato, D. J. Hutchinson, D. Gauthier, and T. Edwards, "Managing rockfall risk through baseline monitoring of precursors using a terrestrial laser scanner," Canadian Geotechnical Journal, vol. 5, no. 7, pp. 953-967, 2017.

[7] M. Barbarella, M. Fiani, and A. Lugli, "Uncertainty in terrestrial laser scanning surveys of landslides," Remote Sensing, vol. 9, pp. 113, 2017.

[8] H. González-Jorge, B. Riveiro, P. Arias, and J. Armesto, "Photogrammetry and laser scanner technology applied to length measurements in car testing laboratories," Measurement, vol. 45, pp. 354363, 2012. 
[9] M. Popia, "Terrestrial laser scanning technology used in the field of shipbuilding," RevCAD Journal of Geodesy and Cadastre, vol. 15, pp. 121-128, 2013.

[10] M. A. Abbas, S. Halim, Z. Majid, A. K. Chong, L. C. Luh, M. F. Mohd Ariff, and K. M. Idris, "Improvement in accuracy for three-dimensional sensor (Faro Photon 120 Scanner)," International Journal of Computer Science Issues, vol. 10, no. 1, pp. 176182, 2013.

[11] P. R. Wolf, B. A. Dewitt, and B. E. Wilkinson, Elements of Photogrammetry with Application in GIS, 4th ed. United State of America: The McGraw-Hill Companies, Inc., 2014.

[12] T. Rabbani, "Automatic reconstruction of industrial installations using point clouds and images," A thesis for the degree of Doctor of Philosophy, TU Delft, 2006.

[13] N. A. Fuad, A. R. Yusoff, M. P. M. Zam, A. Aspuri, M. F. Salleh, Z. Ismail, M. A. Abbas, M. F. Mohd Ariff, K. M. Idris, and Z. Majid, "Evaluating mobile laser scanning for landslide monitoring," in The International Archives of the Photogrammetry, Remote Sensing and Spatial Information Sciences, Volume XLII3/W4, 2018 GeoInformation For Disaster Management (Gi4DM), 18-21 March 2018, Istanbul, Turkey.

[14] F. Remondino, L. Barazzetti, F. Nex, M. Scaioni, and D.Sarazzi, "UAV photogrammetry for mapping and 3D Modeling: current status and future perspectives," in International Archives of the Photogrammetry, Remote Sensing and Spatial Information Sciences, Vol. XXXVIII1/C22 UAV-g 2011, Conference on Unmanned Aerial Vebicle in Geomatics, Zurich, Switzerland, 2012.

[15] P. Martyna and G. François, "Assessing the accuracy of land-based mobile laser scanning data," Geomatics and Environmental Engineering, vol. 6 no. 3, pp. 73-81, 2012.

[16] S. Xu, P. Cheng, Y. Zhang, and P. Ding, "Error analysis and accuracy assessment of mobile laser scanning system," The Open Automation and Control Systems Joumal, vol. 7, no. 1, pp. 485-495, 2015.

[17] N. Darwin, A. Ahmad, ZMat Amin, Z. Majid, M. F. Mohd Ariff, K. A. Idris, M. A. Abbas, and K. Zainuddin, "The accuracy evaluation of unmanned aerial vehicle technology for different coastal terrain mapping," in 2018 8th IEEE International Conference on Control System, Computing and Engineering (ICCSCE 2018), 23-25 November 2018, Penang, Malaysia.

[18] M. A. Abbas, D. D. Lichti, A. K. Chong, S. Halim, and Z. Majid, "An on-site approach for the selfcalibration of terrestrial laser scanner," Measurement, vol. 52, pp. 111-123, 2014.

[19] T. Luhmann, S. Robson, S. Kyle, and I. Harley, Close Photogrammetry: Principles, Methods and Applications. Dunbeath Mains Cottages, Dunbeath, Scotland, United Kingdom: Whittles Publishing, 2006.

[20] L. Zongjian, "UAV for mapping-Low altitude photogrammetric survey," in The International Archives of Photogrammetry, Remote Sensing and Spatial Information Sciences. XXXVII(B1), Beijing, China, 2008.
[21] H. Eisenbeiss, "UAV photogrammetry," Doctor Philosophy, Eidgenössische Technische Hochschule (ETH), Zürich, 2009.

[22] G. Verhoeven, M. Doneus, C. Briese, and F. Vermeulen, "Mapping by matching: A computer vision-based approach to fast and accurate georeferencing of archaeological aerial photographs," Journal of Archaeological Science, vol. 39, no. 7, pp. 20602070, 2012.

[23] D. Dorling and D. Fairbairn, Mapping: Ways of Representing the World. Routledge, 2013.

[24] K. Tahar, "Photogrammetric micro unmanned aerial vehicle for large scale slope mapping," Doctoral Thesis in Geomatics Engineering, University Technology of Malaysia, Malaysia, 2013.

[25] M. A. Abbas, S. Halim, Z. Majid, A. K. Chong, L. C. Luh, K. M. Idris, and M. F. Mohd Ariff, "Terrestrial laser scanners pre-processing: Registration and georeferencing," Jurnal Teknologi UTM, vol. 71, no. 4, pp. 115-122, 2014.

[26] I. Puente, H. González-Jorge, P. Arias, and J. Armesto, "Land-based mobile laser scanning systems: A review," in International Archives of the Photogrammetry, Remote Sensing and Spatial Information Sciences, Volume XXXVIII-5/W12, 2011 ISPRS Calgary 2011 Workshop, 29-31 August 2011, Calgary, Canada.

[27] H. Ali, "Guideline for GNSS calibration using MyRTKnet," Circular Director General of Surveying and Mapping, Department of Surveying and Mapping, Malaysia, 2008.

[28] S. R. Saghravani, S. Mustapha, and S. F Saghravani, "Accuracy comparison of RTK-GPS and automatic level for height determination in land surveying," MASAUM Journal of Reviens and Surveys, vol. 1, no, 1, pp. 10-13, 2009.

[29] C. D. Ghilani and P. R. Wolf, Elementary Surveying: An Introduction to Geomatics, 13th ed. Upper Saddle River, New Jersey: Pearson Education, Inc., 2012.

[30] A. Kukko, H. Kaartinen, J. Hyyppä, and Y. Chen, "Multiplatform mobile laser scanning: Usability and performance," Sensors, vol. 12, pp. 11712-11733, 2012.

[31] S. Dursun, D. Sagir, G. Büyüksalih, and S. Buhur, "3D city modelling of istanbul historic peninsula by combination of aerial images and terrestrial laser scanning data," in XXIst ISPRS Congress, Technical Commission V, ISPRS Archives - Volume XXXVII (Part B7), J. Chen, J. Jiang, and H. G. Maas, Eds. Beijing, China, 2008, pp. 993-998.

[32] M. A. Abbas, S. Halim, Z. Majid, A. K. Chong, K. M. Idris, M. F. Mohd Ariff, A. Aspuri, and A. M. Samad, "Data quality assurance for hybrid and panoramic scanners via self-calibration," in The 6th International Conference on System Engineering and Technology (ICSET2016), October 34, 2016. Institut Teknologi Bandung (ITB), Indonesia. 
[33] C. D. Ghilani, Adjustment Computations: Spatial Data Analysis, 5th ed. Hoboken, New Jersey: John Wiley \& Sons, Inc., 2010.

[34] P. Barry and R. Coakley, "Field accuracy test of RPAS photogrammetry," in International Arcbives of the Photogrammetry, Remote Sensing and Spatial Information
Sciences, Volume XL-1/W2, 4 - 6 September 2013, Rostock, Germany.

[35] J. A. Gonçalves, and R. Henriques, "UAV photogrammetry for topographic monitoring of coastal areas," ISPRS Journal of Photogrammetry and Remote Sensing, vol. 104, pp. 101-111, 2015.

Mohd Azwan Abbas, photograph and biography not available at the time of publication.

Khairulazhar Zainuddin, photograph and biography not available at the time of publication.

Norhadija Darwin, photograph and biography not available at the time of publication.

Mohamad Aizat Asyraff Mohamad Azmi, photograph and biography not available at the time of publication.

Yusuf Drisu Opaluwa, photograph and biography not available at the time of publication.

Saiful Aman Sulaiman, photograph and biography not available at the time of publication.

Norshahrizan Mohd Hashim, photograph and biography not available at the time of publication. 